ISSN: 0514-7336

DOI: http://dx.doi.org/10.14201/zephyrus201677207220

\title{
TESSERAE LUSORIAE EN HISPANIA
}

\section{Tesserae lusoriae in Hispania}

\author{
F. Germán Rodríguez Martín \\ Arqueólogo-exmiembro libre de la Casa de Velázquez. C/ Fuente del Gallo, 24. 11406 Jerez de la Frontera. \\ Correo-e: germanroma@hotmail.com
}

Recepción: 15/07/2015; Revisión: 6/02/2016; Aceptación: 12/04/2016

RESUMEN: Las conocidas como tesserae lusoriae son objetos que han recibido escasa atención por parte de los investigadores hispanos. En la mayoría de los trabajos se ha abordado su estudio de manera tangencial y aislada. A través de las piezas recogidas en el mundo romano, se pueden distinguir varios modelos. En este trabajo abordamos el grupo que cuenta con un mayor número de ejemplares. Se trata de pequeñas fichas realizadas en hueso, y raramente en marfil, de cuerpo rectangular, que rematan en uno de sus extremos en un apéndice circular perforado. En una de las caras llevan grabado un numeral y, en la contraria, calificativos unas veces soeces y otras benévolos. Por el momento se ignora cuál fue su función concreta; de hecho, se han planteado diversas hipótesis sobre la utilidad de estos objetos. La mayoría de los estudiosos consideran que deben estar relacionados con algún tipo de juego del que aún se desconocen las reglas.

Palabras clave: hueso trabajado; juego romano; fichas de juego; inscripción; hallazgos mediterráneos.

ABSTRACT: The known ones as tesserae lusoriae are objects that have received little attention on the part of the Hispanic investigators. Most of the works have boarded its study in a tangential and isolated way. Through the pieces picked up in the Roman world, several models can be distinguished. In this work we approached the group that counts on a greater number of units. One is a small group of cards realized in bone, and rarely in ivory, of rectangular body, which end in one of their ends in a perforated circular appendix. In one of the faces there is an engraving number and in the opposite face there are expressions some of them crude and other benevolent ones. For the moment it is not known which was its concrete function; in fact, diverse hypotheses have considered on the utility of these objects. The majority of the investigators consider that they must be related to some type of game of which we still do not know the rules.

Key words: Worked bone; Roman game; inscription; game cards; Mediterranean findings.

\section{Introducción}

Desde los iniciales trabajos de Blanchet (1889: 225 y ss.) y Huelsen ${ }^{1}$ (1896: 227 y ss.) pocos han sido los

${ }^{1}$ En algunas publicaciones aparece citado como Hülsen, cuando en realidad es Huelsen. En el trabajo publicado en 1896, "Miscellanea epigrafica", Mitteilungen des Kaiserlich estudios centrados en los diversos modelos de las tesserae lusoriae ${ }^{2}$ realizadas en hueso, y raramente en marfil.

Deutschen Archaeologischen Institus, Römische Abteilung, XI, este investigador firma como Huelsen.

${ }^{2}$ Como opina Balil (1987: 334), con el que estamos totalmente de acuerdo, "Estas piezas no son tesserae en su sentido estricto, tampoco son entendidas como sortes propiamente dichas, en cuanto no hay un significado religioso 
En el caso hispano, el primer análisis de conjunto sobre este tipo de piezas fue publicado a finales del s. xx por A. Balil (1987). En ese estudio se recogían únicamente las tesserae procedentes de las excavaciones de Ampurias, exhumadas por el profesor Martín Almagro (1952: 177-180), quien recuperó un conjunto notable. A partir de este estudio, sólo se ha hecho mención de estos objetos en la compilación de Mayer y Rodà (1988: 240-243; IRC, v) ${ }^{3}$. En ella se recogen las tesserae conocidas del área catalana y balear. Posteriormente, Fabre, Mayer y Rodà (2002: 201-204), basándose en el trabajo precedente, vuelven a hacer referencia de forma esporádica, en la obra Inscriptions romaines de Catalogne y en el catálogo de la exposición Scripta manent (Comes y Rodà, 2002: 327). En ambos trabajos se recoge la bibliografía anterior así, como información detallada de las características formales y la cronología.

Recientemente J. M. Abascal (2012: 278-279), al estudiar algunas inscripciones del conventus Carthaginensis, ha llevado a cabo un análisis pormenorizado e interesante de la tessera lusoria procedente de la Colonia Iulia Ilici Augusta (La Alcudia de Elche, Alicante). Poco después G. Baratta (2014: 72-73) ha examinado de forma minuciosa dos tesserae del territorio balear, ya recogidas por Mayer y Rodà (1988: 242-243), una de ellas actualmente perdida ${ }^{4}$ y la otra en la colección de M. Quetglas de Artà (Mallorca).

y su propósito era puramente recreativo". Pese a ello, utilizaremos el termino tesserae lusoriae, pues es el que se emplea asiduamente por la investigación.

3 En esta compilación aparece con el n. ${ }^{\circ} 9.2$ una tessera procedente de Tarrasa, hoy perdida. Fue recogida, según apuntan los autores, en las excavaciones de la iglesia de Santa María. Estuvo conservada en el Museo parroquial, donde desapareció en 1915 (Puig i Cadafalch, 1936: 747, fig. 642; Fabre, Mayer y Rodà, $1984=I R C$ I, n. ${ }^{\circ} 80$, p. 123, lám. XXVII). En una cara aparecía el numeral XXv mientras que la otra parece que estaba en blanco, o no se leyó. Los mismos autores, en un trabajo posterior (Fabre, Mayer y Rodà, 2002), ya no la recogen. Por nuestra parte, al no verla, ni contar en la publicación con la descripción de la pieza, medidas, etc., no la hemos incluido en este trabajo. De ser cierta la lectura, pensamos que podría tratarse de otro modelo de tessera.

4 Sin procedencia conocida en Ibiza (Mayer y Rodà, 1988: 242-243, n. ${ }^{\circ}$ 9.6.1).
Ante esta situación, y aprovechando la revisión que estamos llevando a cabo de las piezas talladas en hueso de época romana en Hispania ${ }^{5}$, hemos decidido abordar el tema actualizando los hallazgos conocidos y revisando la información disponible, con la intención de ofrecer algunos datos que puedan contribuir a desentrañar cuál pudo ser la verdadera función de estas piezas.

\section{Características de las tesserae lusoriae hispanas en hueso}

Dentro de esta categoría se engloban varios modelos de fichas de juego. Las que aquí presentamos están fabricadas en hueso, aunque también las hubo en marfil. Son pequeños objetos de forma rectangular que rematan en uno de sus extremos en un apéndice circular. A partir de estas características formales hemos podido localizar, hasta el momento, en la Península Ibérica más de 21 piezas, que hemos reunido en varios grupos que presentan patrones bien diferenciados ${ }^{6}$. En este primer avance del estudio, nos hemos centrado únicamente en las que hemos clasificado como grupo 1, las tesserae lusoriae que llevan numeral e inscripción 7 . Este grupo es el que cuenta, hasta el momento, con el mayor número de muestras conocidas en Hispania.

\section{Téseras con numeral e inscripción}

El modelo de tessera lusoria al que nos referimos -no muy habitual entre las conocidas en el mundo

5 Tenemos en prensa un volumen dedicado a los materiales de hueso trabajado de época romana en Hispania. En dicho estudio abordamos el resto de modelos de tesserae localizados en la Península Ibérica encuadrados en el grupo RM.E.III.1.

${ }^{6}$ Caso de la recogida en Ampurias con el n. ${ }^{\circ} 134$ por Almagro (1952: 178) y el n.o 164 de Fabre, Mayer y Rodà (2002: 202) que, como ya apreció Balil (1987: 333), debía corresponder a un grupo diferente. Dicha tessera lleva en una cara el numeral xxiıI y el reverso está liso. Fabre, Mayer y Rodà plantean que, en caso de estar grabada, debería llevar el nombre de Fortunate. En nuestra opinión no hubo intención de poner ningún nombre, pues pertenece a un segundo grupo de nuestra clasificación (RM.III.E.1.2) que únicamente llevan numeral.

7 Se engloba en el grupo RM.III.E.1.1. 
romano- responde a pequeñas fichas, elaboradas en hueso, de cuerpo rectangular, tipo bastoncillo, que remata en uno de los extremos en un apéndice circular. Dicho botón presenta una decoración, más o menos profusa, de círculos concéntricos incisos. Esta protuberancia está perforada en sentido transversal (Cecchini, 2015: 123-124). En la mayoría de los casos, el campo epigráfico ha sido delimitado mediante líneas incisas en los extremos; sólo excepcionalmente puede estar delimitado en los cuatro lados por líneas rectas.

Respecto a las dimensiones, destaca claramente el hecho de que las medidas suelen ser muy similares, con ligeras diferencias en el volumen del cuerpo. Tienen una longitud que oscila entre 5,6 y 4,7 $\mathrm{cm}$; en ocasiones nos encontramos con algunas piezas de menor tamaño $-4,1 \mathrm{~cm}$ - debido a la pérdida del apéndice y a su posterior reutilización. En estos casos, se ha recurrido a volver a hacer un orificio en la zona del campo epigráfico, con toda probabilidad por la fractura del apéndice inicial ${ }^{8}$. La anchura total de la ficha fluctúa entre 0,6 y $1,1 \mathrm{~cm}$, siendo la medida más frecuente la de $0,9 \mathrm{~cm}$. El grosor en general suele estar entre 0,5 y $0,7 \mathrm{~cm}$, aunque la más utilizada corresponde a $0,5 \mathrm{~cm}$.

La característica que define el grupo que aquí presentamos, aparte de la perforación en la prominencia, es precisamente que las dos caras siempre aparecen ocupadas: una por un numeral y la contraria por un apelativo. Normalmente para el proceso de grabado de la inscripción se ha recurrido a la letra capital.

El contenido epigráfico suele recoger expresiones tanto de tipo laudatorio como soeces. Hasta la fecha se han constatado en el mundo romano 57 términos diferentes, que podrían quedar reducidos a 55, si tenemos en cuenta que 2 de ellos corresponden al mismo vocablo con ligeras variantes. En relación con los numerales grabados, hay que reseñar que son cifras que abarcan desde el I al xL, observándose un salto entre el xxx y el XL.

${ }^{8}$ Un ejemplo es la tessera lusoria ampuritana de Amator (Almagro, 1952: 177, n. ${ }^{\circ} 131$ ); n. ${ }^{\circ} 1612$ en inventario del MAC. Barcelona. Se ha reaprovechado haciéndole una perforación en el inicio del campo epigráfico.
La cronología que se les viene asignando a tenor de los hallazgos se emplaza entre los ss. II y i a. C., situándose su momento álgido en la etapa tardorrepublicana, datación que también se le asigna a las tesserae hispanas (Almagro, 1952: 177 y ss.; Mayer y Rodà, 1988: 243; Corel, 2002: 396; Abascal, 2012: 279; Baratta, 2014: 70). Esta cronología deriva fundamentalmente de los contextos de la necrópolis de Perugia (Huelsen, 1896: 227 y ss.) y de la cisterna de Vesta (Campagna, 1995: 215 y ss.; De Grossi, 2011: 136-137). De momento, no se ha constatado su pervivencia en época imperial.

En cuanto a su procedencia, hay que señalar que se han encontrado algunas piezas de este tipo fabricadas en hueso, y raramente en marfil, muy diseminadas por diversos puntos de la península itálica (Huelsen, 1896: 227-237; Campagna, 1995: 262263 y 283-288; De Grossi, 2011: 136-137; Casagrande, 2014: 247-252; Cechini, 2015: 67-70) y, en menor medida, en Hispania (Almagro, 1952: 177-180; Balil, 1987: 331-336; Mayer y Rodá, 1988: 240-243; Fabre, Mayer y Rodà, 2002: 201 204; Abascal, 2012: 278-279; Beltrán, 1983: 52; Castello, 1998: 74-76; Veny, 1965: 71 y 223-224; Baratta, 2014: 69-74). Si prestamos atención a los lugares donde se concentra el mayor número de piezas conocidas, da la impresión de que su ámbito de mayor aceptación se localiza en la región meridional de Italia. De hecho, la presencia de estas piezas es importante en el área napolitana. Esto nos lleva a plantear la posibilidad de que desde esta zona, con la que existieron importantes conexiones comerciales, pasase al área mediterránea de la Península Ibérica. No obstante, también se constatan tanto en otros puntos de la península italiana, como en otras provincias imperiales, aunque en menor cantidad.

Resulta obligado mantener cierta cautela respecto a los lugares en los que hoy se localizan estos ejemplares. Debemos tener presente que un buen número de estos objetos forman parte de colecciones particulares que no siempre proceden de hallazgos cercanos. En algunos casos se trata de conjuntos adquiridos por coleccionistas, a lo largo de los años, en los mercados de antigüedades. De hecho, hemos podido comprobar que en algunos casos el objeto adquirido circula por varias manos, pasando incluso 


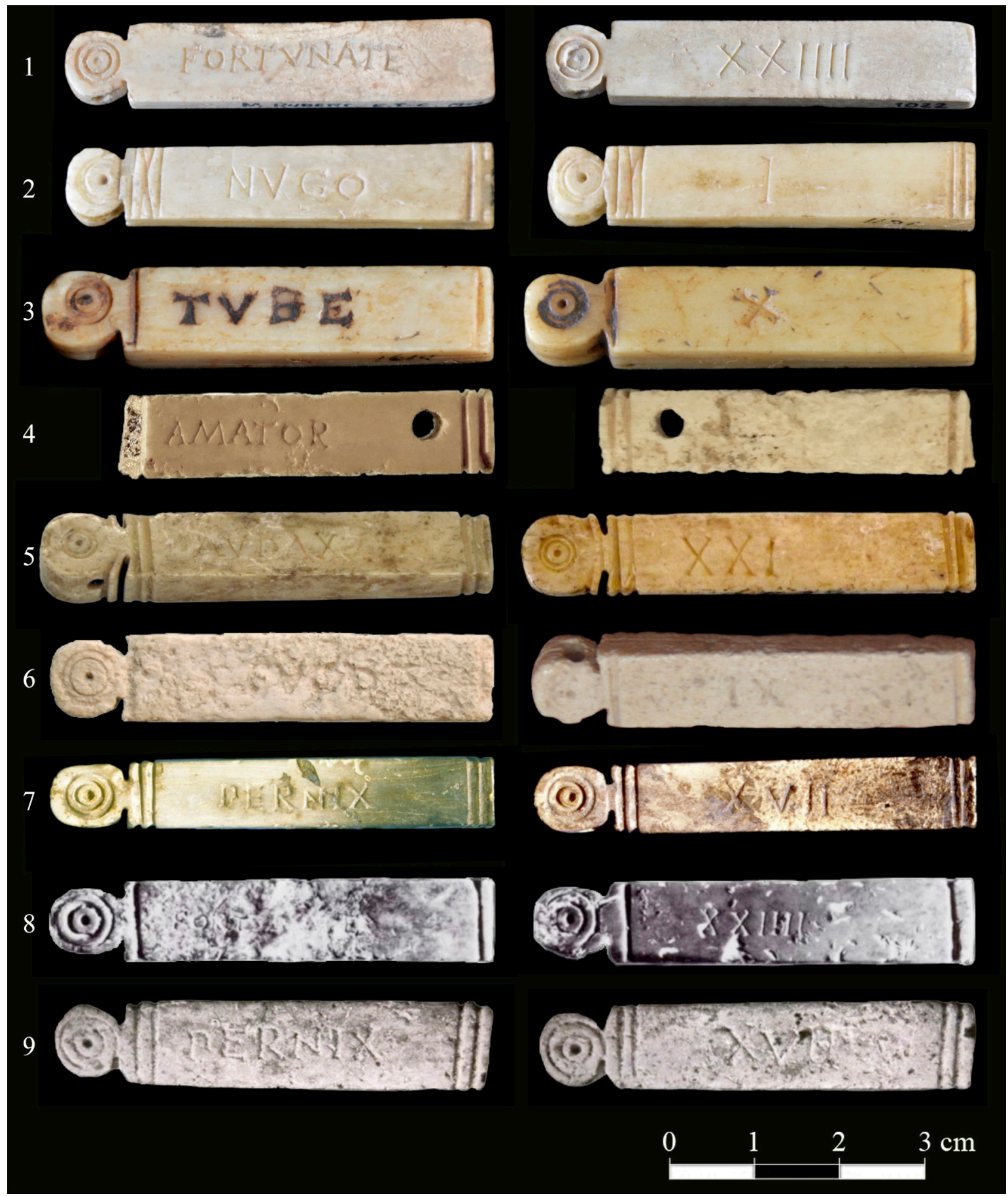

Fig. 1. Tesserae lusoriae del grupo I encontradas en la Peninsula Ibérica, con su correspondiente leyenda, lugar de hallazgo y localización actual: 1) FORTVNATE (Emporiae; MAC Ampurias); 2) NVGO (Emporiae; MAC Barcelona); 3) TVBE (Emporiae; MAC Barcelona); 4) AMATOR (Emporiae; MAC Barcelona; imagen cortesía del MAC Barcelona); 5) AVDAX (Emporiae; MAC Gerona; imagen cortesía del MAC Gerona); 6) ----- IX (Emporiae; M. Prehistoria de Valencia); 7) PERNIx (Illici; Museo de la Alcudia, Elche; imagen cortesía de J. M. Abascal); 8) fortv[n]ATE/Xximi (Saguntum; Col. Torres; imagen tomada de Corell, 2002: p. 396; 9) PERnix/XviI (Talamanca, Ibiza; Col. particular; imagen tomada de Baratta, 2014: 71, fig. 1). 
de un país a otro 9 . Con este panorama resulta muy difícil, salvo las exhumadas en excavaciones, conocer su procedencia y poder hacer una valoración de los lugares de hallazgo.

Actualmente, en Hispania, hemos podido compilar 12 fichas $^{10}$ (Fig. 1), cuyos lugares de procedencia se circunscriben, casi en exclusividad, al área costera catalano-levantino-balear (Fig. 8). Concretamente 7 de ellas han aparecido en la ciudad de Emporiae (Almagro, 1952: 177-180; Balil, 1987: 334-335), 1 en Dertosa (Albertini, 1918: 330-331; Mayer y Rodà, 1988: 240-243; Fabre, Mayer y Rodà, 2002: 204) y las 4 restantes se reparten: 1 en Saguntum (Corell, 2002: 396; CIL II $^{2} / 14$, 579a) ${ }^{11}$, 2 en Baleares (Fita, 1907: 354; Beltrán, 1983: 52; Mayer y Rodà, 1988: 242-243; Castelló, 1988: 74-76; Veny, 1965: 71 y 223-224; Baratta, 2014: 72-73) y 1 en Ilici (Abascal, 2012: 278-279). La justificación de lo que parece una buena acogida en esta zona peninsular, y no en otras áreas del territorio hispano, habría que ponerla en relación con sus propios orígenes. El nexo de unión entre el $s$ de Italia y el área catalano-levantina se encuentra en que sus bases históricas enraízan en la colonización griega. Consideramos que esta no es una cuestión baladí, sobre todo si tenemos en cuenta que ya existieron con anterioridad fichas escritas en griego con iguales características portando un numeral y un calificativo (Huelsen, 1896: 238 y ss.). La misma opinión es mantenida por G. Baratta (2014: 70), quien plantea que pudo tratarse de un juego importado desde Italia que desaparece en época imperial.

9 Es el caso, por ejemplo, de la tesserae lusoriae de Fur $r$ - de, de la colección Hernández de Tarragona, adquirida en Italia, sin que sepamos su procedencia (Huelsen, 1896: 233) CIL II, 4968, 2.

${ }^{10}$ No incluimos la tessera de la colección Hernández, de Tarragona, cit. n. 9, por ser una pieza procedente de Italia. Tampoco la procedente de Tarrasa con el numeral xxv, recogida por Mayer y Rodá (1988: 241, n. ${ }^{\circ}$ 9.2) por los argumentos expresados anteriormente en la n. 3 .

11 Alföldy (1995: 114, no 14, 579a) transcribe esta tessera con el numeral XiıI/Fortunate, cuando en realidad, como se puede ver en la fotografía presentada por Corell (2002: 396), el numeral es XximI (Fig. 1).
Tanto las tesserae hispanas como las italianas obedecen al mismo patrón, presentando en una cara un calificativo y en la otra un numeral. Esta es la diferencia fundamental respecto a las tesserae nummulariae que, aunque comparten similitud formal que podría conducir a equívocos, al analizarlas detenidamente se observa de forma clara que corresponden a una serie distinta. En estas últimas, las expresiones, que suelen ocupar las cuatro caras de la ficha, hacen referencia a servi, domini, personajes públicos, etc., e incluso, en ocasiones, suelen alternar en alguna de sus caras con elementos vegetales ${ }^{12}$.

\subsection{Sobre su posible funcionalidad}

En realidad, no sabemos cuál fue su función. Se han planteado varias hipótesis sobre su utilidad. En un primer momento, Huelsen (1896: 227 y ss.) opina que puede tratarse de fichas vinculadas a las representaciones teatrales, aunque con posterioridad piensa que habría que relacionarlas con el ludus duodecim scriptorium, a partir de la presencia del término Moraris. Es Balil (1987: 334-335) quien las pone en sintonía con juegos relacionados con el azar. Más adelante, Pedroni (1995: 182-183) comienza a relacionarlas con un juego militar de control, para posteriormente decantarse por un juego de azar. La misma interpretación es la proporcionada por Gostenčnik y Deschler-Erb (2008: 293) y más recientemente por Cecchini (2015: 67). Casagrande (2012: 251-252) las vincula con la suerte. Considera que pudo consistir en un sistema simple de asociación de la denominación de un grupo y su emparejamiento de modo casual, como el juego del tarot o de las runas.

Por el contrario, otros investigadores las incluyen dentro del bloque de las tesserae nummulariae. Plantean que pudieron ser usadas como etiquetas dentro del sistema bancario romano, en el que las

12 Ejemplo de este tipo de piezas son: las depositadas en Francia en el Cabinet des Médailles, procedentes de la colección Froehner, quien las adquirió en Roma a principios del s. $\mathrm{xx}$ (Andreau, 2001: 331 y ss.); las estudiadas por Pedroni (1995: 162-178) para Italia, o las de Magdalensberg (Gostenčnik, 2005: 253-258; Deschler-Erb-Gostenčnik, 2008: 293). Todas ellas corresponden con tesserae nummulariae. 


\begin{tabular}{|c|c|c|c|c|}
\hline DENOMINACIÓN & CIFRA & SIGNIFICADO & LUGAR DE HALLAZGO & LOCALIZACIÓN ACTUAL \\
\hline FORTVNATE & XXIIII & afortunado & Emporiae & MAC Ampurias \\
\hline AMATOR & -.--- & amante/mujeriego & Emporiae & MAC Barcelona \\
\hline$N V G O$ & $\mathrm{I}$ & bromista/impostor & Emporiae & MAC Barcelona \\
\hline TVBE & $\mathrm{x}$ & sodomita & Emporiae & MAC Barcelona \\
\hline$A V D X$ & XXI & audaz & Emporiae & MAC Girona \\
\hline$C L----$ & $\mathrm{x}$ & --.---.-- & Emporiae & MAC Girona \\
\hline $\begin{array}{l}----- \\
----\end{array}$ & IX & --.----- & Emporiae & M. Prehistoria de Valencia \\
\hline PERNIX & XVII & ágil/veloz/infatigable & Illici & M. Alcudia de Elche \\
\hline PERNIX & XVII & ágil/veloz/infatigable & Talamanca (Ibiza) & Col. particular \\
\hline PERNIX & XVII & ágil/veloz/infatigable & Pollentia & Col. particular, M. Quetglas \\
\hline FORTVNATE & XXIIII & afortunado & Saguntum & Col. particular, J. M. Torres \\
\hline FORTVNAT(VS) ${ }^{13}$ & {$[\mathrm{x}]$ XIIII } & afortunado & Dertosa & Col. particular Mestre i Noé \\
\hline
\end{tabular}

FIG. 2. Relación de tesserae lusoriae hispanas con su procedencia y contenido.

cifras hacen referencia a monedas (Andreau, 2001: 332). Gamurrini (1887: 236) y De Grossi (2011: 137) las consideran fichas de un juego similar a la lotería, o bien de un pasatiempo parecido a los naipes.

Resulta evidente que hasta la fecha cualquier hipótesis sobre el uso de estos objetos puede ser válida. Pensamos que se trata de una distracción relacionada con el azar, cuyas reglas, en palabras de Abascal (2012: 278), son "difíciles de conocer".

Lo que sí parece cierto, a tenor de la perforación transversal, es que este orificio servía para unir las piezas mediante un cordón. De este modo, se podían

13 No hemos podido ver la tessera lusoria recogida por Albertini (1918: 330-31), depositada en la colección Mestre y Noé de Tortosa. Nos llama la atención que aparezca el término completo, Fortunatus, cuando en el resto de las conocidas aparece Fortunatle; lo mismo sucede con el numeral que Albertini consigna como VIIII. No sabemos si este investigador llegó a ver la pieza o simplemente le proporcionaron información con las medidas, pues en su artículo no adjunta ni dibujo ni fotografía. Pensamos, sobre todo por la cifra, que al menos la cara del numeral debe estar dañada. Lo planteamos porque esta expresión, en los seis casos conocidos, siempre aparece vinculada al número XXIIII. Es posible que lo que haya copiado como $v$ en realidad corresponda a la parte superior de una $\mathrm{x}$ (por estar dañada la pieza), faltando otra $\mathrm{x}$ anterior, como ha ocurrido en la tessera de Saguntum (n. 11). Lo mismo pudo pasar con el nombre que, tal vez, al aparecer como Fortunat, lo reprodujese como Fortunat(us), pensando más en que se trataba de un nombre que de una expresión (afortunado). De ahí que nos inclinemos por la opción Fortunat/XXIIII. tanto trasladar como guardar sin riesgo de perderse. No creemos que se utilizasen estando todas enlazadas. Pensamos, al igual que Balil (1987: 334), que, tal vez, se depositaban en una bolsa, de donde se sacarían de forma individualizada. Una vez concluido el juego se volverían a reunir con el cordón.

Un análisis de todas las tesserae lusoriae conocidas hasta el momento nos permite extraer algunas conclusiones que tal vez puedan ayudar a comprender un poco mejor este pasatiempo.

Como hemos señalado con anterioridad, el conjunto más importante de este modelo de fichas procede de la necrópolis de Perugia (Huelsen, 1896: 227 y ss.) y de la cisterna de Vaste (Campagna, 1995: 215 y ss.; De Grossi, 2011: 136-137). El resto de los hallazgos corresponde a ejemplares aislados o en escaso número. La mayor recopilación publicada hasta el día de hoy es la de Huelsen (1896), que recoge 84 fichas procedentes de museos y colecciones particulares repartidas por Europa. Al final de su estudio ofrece un listado de las palabras y cifras identificadas. Llega a recopilar una seriación de cuarenta números a los que se le asocian sistemáticamente diferentes nombres. Observa que consta de treinta números entre el I y el xL, en los que faltan los comprendidos entre el Xxx y XL.

Recientemente Casagrande (2012), a partir del estudio de unas tesserae de Perugia, ha puesto al día la relación de nombres y números de las piezas contabilizadas en los últimos ańos. En dicho estudio no 


\begin{tabular}{|c|c|c|c|c|c|c|c|}
\hline DENOMINACIÓN & \multicolumn{6}{|c|}{ NuMERALES Y LUGARES DE HALLAZGO } & CIFRAS Y N.o DE PIEZAS $^{14}$ \\
\hline AMATOR & $\begin{array}{c}----- \\
(\mathrm{AP})\end{array}$ & $\begin{array}{l}---- \\
(\mathrm{MT})\end{array}$ & $\begin{array}{r}\mathrm{XXX} \\
(\mathrm{PE})\end{array}$ & $\begin{array}{c}\mathrm{XX} \\
(\mathrm{RM})\end{array}$ & $\begin{array}{l}\mathrm{XXX} \\
(\mathrm{DE})\end{array}$ & $\begin{array}{l}\text { XIIII } \\
(\mathrm{VA})\end{array}$ & $30(3)-\mathrm{I} 4(\mathrm{I})$ \\
\hline ARGVTE & $\begin{array}{l}\mathrm{XV} \\
(\mathrm{PE})\end{array}$ & $\begin{array}{c}\mathrm{XV} \\
(\mathrm{NC})\end{array}$ & $\begin{array}{c}\mathrm{XV} \\
(\mathrm{AM})\end{array}$ & & & & I $5(3)$ \\
\hline ARPAX & $\begin{array}{l}\text { XIX } \\
\text { (св) }\end{array}$ & $\begin{array}{l}\text { XVIIII } \\
(\mathrm{PT})\end{array}$ & $\begin{array}{l}\text { XVIIII } \\
\text { (DR) }\end{array}$ & $\begin{array}{l}\text { XIIII } \\
(\mathrm{DE})\end{array}$ & & & I9 $(3)-$ I $4(\mathrm{I})$ \\
\hline$A V D A X$ & $\begin{array}{l}\text { XXI } \\
(\mathrm{AP})\end{array}$ & $\begin{array}{l}\text { XXI } \\
(\mathrm{AN})\end{array}$ & & & & & $2 \mathrm{I}(2)$ \\
\hline AVIDVS & $\begin{array}{c}\mathrm{XVIII} \\
(\mathrm{DE})\end{array}$ & $\begin{array}{l}\mathrm{XV} \\
(\mathrm{VA})\end{array}$ & & & & & I $8(\mathrm{I})-\mathrm{I} 5(\mathrm{I})$ \\
\hline$B O N V S$ & $\begin{array}{l}\text { XVII } \\
(\mathrm{VA})\end{array}$ & & & & & & I7 \\
\hline BENIGNVS & $\begin{array}{c}\mathrm{XX} \mathrm{A} \\
(\mathrm{PE})\end{array}$ & $\begin{array}{r}\mathrm{Xx} \mathrm{A} \\
(\mathrm{LV})\end{array}$ & & & & & $20 \mathrm{~A}(2)$ \\
\hline BENIGNE & $\begin{array}{c}\mathrm{XXX} A \\
(\mathrm{CB})\end{array}$ & & & & & & $30 \mathrm{~A}(\mathrm{I})$ \\
\hline BENEST & $\begin{array}{l}\mathrm{xxV} \\
(\mathrm{vA})\end{array}$ & & & & & & $25(\mathrm{I})$ \\
\hline CATV... & $\begin{array}{l}\mathrm{v} \\
(\mathrm{cs})\end{array}$ & & & & & & $5(\mathrm{I})$ \\
\hline $\begin{array}{l}\text { CINAIDVS/ } \\
\text { CINAEDVS }\end{array}$ & $\begin{array}{l}\text { VII } \\
(\mathrm{CU})\end{array}$ & $\begin{array}{l}\text { VII } \\
(\mathrm{AN})\end{array}$ & & & & & $7(2)$ \\
\hline$C L \ldots$ & $\frac{\mathrm{xx}-}{(\mathrm{AP})}$ & & & & & & $20(\mathrm{I})$ \\
\hline CVNV. LINGE & $\begin{array}{l}\mathrm{VI} \\
(\mathrm{PO})\end{array}$ & $\begin{array}{l}\text { VIII } \\
(\mathrm{NF})\end{array}$ & & & & & $6(\mathrm{I})-8(\mathrm{I})$ \\
\hline CVNNIO & $\begin{array}{c}\mathrm{X} \\
(\mathrm{PT})\end{array}$ & & & & & & Io $(\mathrm{I})$ \\
\hline EBRIOSE & $\begin{array}{l}\text { IIII } \\
(\mathrm{PO})\end{array}$ & $\begin{array}{l}\mathrm{IIII} \\
(\mathrm{RM})\end{array}$ & $\begin{array}{l}\text { IIII } \\
\text { (RV) }\end{array}$ & $\begin{array}{c}\text { IIII } \\
(\mathrm{RV})(\mathrm{VA})\end{array}$ & & & $4(4)$ \\
\hline (F) ACETE & $\begin{array}{l}\mathrm{IIII} \\
(\mathrm{PE})\end{array}$ & $\begin{array}{c}\text { XI } \\
\text { PAL (sC) }\end{array}$ & $\begin{array}{l}\mathrm{XXV} \\
\text { (INC) }\end{array}$ & $\begin{array}{l}\mathrm{XV} \\
\text { (PS) }\end{array}$ & & & $4(\mathrm{I})-\mathrm{II}(\mathrm{I})-25(2)$ \\
\hline FATVE & $\begin{array}{c}\mathrm{XI} \\
(\mathrm{CG})\end{array}$ & $\begin{array}{l}\text { XIIII } \\
(\mathrm{RV})\end{array}$ & $\begin{array}{l}\text { XVII } \\
\text { (BR) }\end{array}$ & $\mathrm{xX}$ A (vi) & & & II I I $)-\mathrm{I} 4(\mathrm{I})-\mathrm{I} 7(\mathrm{I}) 20 \mathrm{~A}(\mathrm{I})$ \\
\hline FELIX & $\begin{array}{l}\mathrm{LX} \\
(\mathrm{PE})\end{array}$ & $\begin{array}{c}\mathrm{LX} \\
\text { (INC) }\end{array}$ & $\begin{array}{c}\text { XXII }^{15} \\
\text { (LO) }\end{array}$ & $\begin{array}{l}\text { XXII } \\
(\mathrm{DE})\end{array}$ & $\begin{array}{l}\mathrm{xxx} \\
(\mathrm{vA})\end{array}$ & $\mathrm{LX}^{16}(\mathrm{CS})$ & I $2(\mathrm{I})-3 \mathrm{O}(\mathrm{I})-40(3)$ \\
\hline
\end{tabular}

${ }^{16}$ Cecchini (2015: 70, n. ${ }^{\circ}$ 6) considera que el numeral

${ }^{14}$ La primera cifra corresponde con el numeral. Entre paréntesis, el número de veces que aparece.

15 Hemos excluido la pieza publicada por Pedroni, ya que no tiene las mismas características que las estudiadas aquí; además, su lectura no es clara, mientras que la numeración considerada es XXII creemos que debería ser XXX. retrógrado corresponde al xxII, visión que no compartimos. Nos basamos en que al tratarse de una numeración retrógrada, al transcribirla podríamos forzar y leer XIII, pero nunca el número XXII propuesto por Cecchini. Ahora bien, si tenemos en cuenta la frecuencia del número asociado a la expresión Felix, en las piezas recogidas por Huelsen, nos parece más apropiado asociarlo al numeral LX. 


\begin{tabular}{|c|c|c|c|c|c|c|c|c|}
\hline DENOMINACIÓN & & & Nume & ESS Y LUC & RES DE HALLAZGO & & & CIFRAS Y N. $0^{\circ}$ DE PIEZAS \\
\hline FICOSE & $\begin{array}{l}\text { VII } \\
(\mathrm{BA})\end{array}$ & $\begin{array}{l}\mathrm{XIV} \\
(\mathrm{CR})\end{array}$ & $\begin{array}{c}\mathrm{v} \\
(\mathrm{VA})\end{array}$ & & & & & $5(\mathrm{I})-7(\mathrm{I})-\mathrm{I} 4(\mathrm{I})$ \\
\hline FORTVNAT/E & $\begin{array}{c}\text { XXIIII } \\
(\mathrm{AP})\end{array}$ & $\begin{array}{c}\text { XXIIII } \\
(\mathrm{cs})\end{array}$ & $\begin{array}{c}\text { XXIIII } \\
(\mathrm{RV})\end{array}$ & $\begin{array}{c}\text { XXIIII } \\
(\mathrm{FL})\end{array}$ & $\begin{array}{c}\mathrm{XXIIII}^{17} \\
(\mathrm{sG})\end{array}$ & $\begin{array}{c}\text { XXVIIII }^{18} \\
(\mathrm{TOR})\end{array}$ & & $24(6)$ \\
\hline FVVCO & $\begin{array}{c}--\mathrm{A}^{19} \\
(\mathrm{PE})\end{array}$ & $\begin{array}{l}\mathrm{XIIA} \\
(\mathrm{PT})\end{array}$ & & & & & & I2 A (2) \\
\hline $\begin{array}{l}F V R / \\
F V R R--D E^{20}\end{array}$ & $\begin{array}{l}\text { II A } \\
\text { (INC) }\end{array}$ & $\begin{array}{c}\mathrm{II} \\
(\mathrm{NC})\end{array}$ & $\begin{array}{l}\text { II A } \\
\text { (PT) }\end{array}$ & $\begin{array}{c}\mathrm{III} \\
(\mathrm{MB})\end{array}$ & $\begin{array}{l}\mathrm{XIII} \\
(\mathrm{VA})\end{array}$ & $\begin{array}{l}\text { II A }{ }^{21} \\
\text { (TA) }\end{array}$ & & $2 A(3)-2(I)-3(I)-I 3(I)$ \\
\hline GAVDESNE & $\begin{array}{c}\mathrm{XI} \\
(\mathrm{DR})\end{array}$ & $\begin{array}{c}\mathrm{XI} \\
(\mathrm{AN})\end{array}$ & & & & & & II $(2)$ \\
\hline GVLO & $\begin{array}{l}\text { IIII } \\
(\mathrm{CG})\end{array}$ & & & & & & & $4(1)$ \\
\hline GVMIA & $\begin{array}{l}\mathrm{XX} \\
(\mathrm{RV})\end{array}$ & $\begin{array}{l}\mathrm{XXI} \\
(\mathrm{PT})\end{array}$ & & & & & & $20(1)-21(1)$ \\
\hline INFELLIX & $\begin{array}{l}\text { IIII } \\
\text { (LO) }\end{array}$ & & & & & & & $4(1)$ \\
\hline INPVDES & $\begin{array}{l}\text { XXII } \\
(\mathrm{DR})\end{array}$ & & & & & & & $22(1)$ \\
\hline LVPA & $\begin{array}{l}\mathrm{XVI} \\
(\mathrm{RV}) \\
\end{array}$ & $\begin{array}{l}----- \\
(\mathrm{BC}) \\
\end{array}$ & $\begin{array}{l}\mathrm{XIA} \\
(\mathrm{HE}) \\
\end{array}$ & & & & & $11 \mathrm{~A}(1)-16(1)$ \\
\hline MALEST & $\begin{array}{l}\text { XXIII A } \\
\text { (BM) }\end{array}$ & & & & & & & 23 A (1) \\
\hline MOICE & $\begin{array}{l}\mathrm{III} \\
(\mathrm{PE})\end{array}$ & $\begin{array}{l}\text { III A } \\
\text { (DR) }\end{array}$ & $\begin{array}{l}\text { III } \\
(\mathrm{Cs})\end{array}$ & & & & & $3(2)-3$ A $(1)$ \\
\hline MOECE & $\begin{array}{l}\text { XXIII } \\
(\mathrm{MT})\end{array}$ & & & & & & & $23(1)$ \\
\hline MORARIS & $\begin{array}{r}\mathrm{XIIII} \\
(\mathrm{PE}) \\
\end{array}$ & & & & & & & $14(1)$ \\
\hline MVLA & $\begin{array}{l}\mathrm{VI} \\
\text { (DR) }\end{array}$ & $\begin{array}{l}\mathrm{VI} \\
(\mathrm{HE})\end{array}$ & & & & & & $6(2)$ \\
\hline NVGATOR & $\begin{array}{c}\mathrm{I} \\
(\mathrm{NC})\end{array}$ & $\begin{array}{c}\mathrm{XI} \\
(\mathrm{BC})\end{array}$ & & & & & & I (I) - I I (I) \\
\hline NVGOR & $\begin{array}{c}\mathrm{I} \\
(\mathrm{AP}) \\
\end{array}$ & $\begin{array}{c}\mathrm{I} \\
\text { (LO) }\end{array}$ & $\begin{array}{c}\mathrm{I} \\
(\mathrm{VA}) \\
\end{array}$ & & & & & I $(3)$ \\
\hline PATICE $/ N$ & $\begin{array}{l}\text { VIII } \\
(\mathrm{PE}) \\
\end{array}$ & $\begin{array}{l}\mathrm{VIII} \\
(\mathrm{CH}) \\
\end{array}$ & $\begin{array}{l}\mathrm{VIII} \\
(\mathrm{vA}) \\
\end{array}$ & $\begin{array}{l}\mathrm{VII} \\
(\mathrm{RV})\end{array}$ & & & & $8(3)-7(\mathrm{I})$ \\
\hline PERNIX & $\begin{array}{r}\mathrm{XVII} \\
(\mathrm{PE})\end{array}$ & $\begin{array}{l}\text { XVII } \\
\text { (INC) } \\
\end{array}$ & $\begin{array}{l}\mathrm{xVII} \\
(\mathrm{cu})\end{array}$ & $\begin{array}{l}\text { XVII } \\
(\mathrm{NF}) \\
\end{array}$ & $\begin{array}{c}\text { XVII } \\
\text { (AC) }(\mathrm{EL})(\mathrm{TAL}) \\
\end{array}$ & $\begin{array}{c}\text { XVIII } \\
(\mathrm{CS})\end{array}$ & $\begin{array}{l}\text { XVII } \\
(\mathrm{RV})\end{array}$ & I7 (9) \\
\hline PERIVRE & $\begin{array}{l}\mathrm{IIII} \mathrm{A} \\
(\mathrm{vA})\end{array}$ & & & & & & & $4 \mathrm{~A}(\mathrm{I})$ \\
\hline
\end{tabular}

17 Cf. n. 11.

18 Cf. n. 12. Consideramos que el numeral debe ser XxiIII.

19 Al estar dañado, creemos que el número que falta podría ser el XII, en base al nombre y cifra aparecida en otro ejemplar.
${ }^{20}$ La lectura completa debe ser Fur r[i]de.

21 La pieza fue adquirida en Italia, sin que sepamos a ciencia cierta en qué lugar; actualmente está integrada en la colección Hernández de Tarragona. 


\begin{tabular}{|c|c|c|c|c|c|c|c|c|}
\hline DENOMINACIÓN & \multicolumn{7}{|c|}{ NUMERALES Y LUGARES DE HALLAZGO } & CIFRAS Y n.o DE PIEZAS \\
\hline PIGER & $\begin{array}{c}---- \\
(\mathrm{PE})\end{array}$ & & & & & & & $5(\mathrm{I})$ \\
\hline$P V L$ & $\begin{array}{c}\mathrm{XX} \\
(\mathrm{DE})\end{array}$ & & & & & & & $20(\mathrm{I})$ \\
\hline PVRVS & $\begin{array}{l}\text { XIIX } \\
\text { (VA) }\end{array}$ & & & & & & & I 8 (I) \\
\hline POPINO & $\begin{array}{l}\text { XIII } \\
\text { (LO) }\end{array}$ & & & & & & & I3 (I) \\
\hline REX & $\begin{array}{c}\mathrm{XL} \\
(\mathrm{VA})\end{array}$ & & & & & & & $40(\mathrm{I})$ \\
\hline SCVLTVMO & $\begin{array}{l}\text { VIIII } \\
\text { (VA) }\end{array}$ & & & & & & & $9(\mathrm{I})$ \\
\hline SERIVS & $\begin{array}{l}\mathrm{XIX} \\
(\mathrm{VA})\end{array}$ & & & & & & & I9 (I) \\
\hline STVMACOSE & $\begin{array}{l}\text { II A } \\
\text { (BM) }\end{array}$ & & & & & & & $2 \mathrm{~A}(\mathrm{I})$ \\
\hline$T V B E$ & $\begin{array}{l}{ }^{22} \\
(\mathrm{PE})\end{array}$ & $\begin{array}{c}\mathrm{X} \\
(\mathrm{AP})\end{array}$ & $\begin{array}{c}\mathrm{x} \\
(\mathrm{cs})\end{array}$ & $\begin{array}{c}\mathrm{x} \\
(\mathrm{VA})\end{array}$ & & & & Io $(3)$ \\
\hline TRICO & $\begin{array}{c}\mathrm{XI} \\
(\mathrm{KE})\end{array}$ & $\begin{array}{l}\mathrm{XI} A \\
\text { (vA) }\end{array}$ & & & & & & I I (I) I I A (I) \\
\hline VAPIO & $\begin{array}{l}\text { IIII } \\
\text { (PO) }\end{array}$ & $\begin{array}{l}\text { IV A } \\
\text { (во) }\end{array}$ & VIII A (LV) & $\begin{array}{c}\text { VIII A } \\
\text { (PT) }\end{array}$ & $\begin{array}{c}\text { VIII A } \\
(\mathrm{KE})\end{array}$ & $\begin{array}{l}\text { VIII A } \\
\text { (NC) }\end{array}$ & $\begin{array}{r}\text { IV A } \\
\text { (VI) }\end{array}$ & $4(\mathrm{I})-4 \mathrm{~A}(2)-8$ A (4) \\
\hline$V E L$ & $\begin{array}{l}\mathrm{XXX} \\
(\text { во) }\end{array}$ & $\begin{array}{l}\mathrm{XXX} \\
(\mathrm{Cs})\end{array}$ & & & & & & $30(2)$ \\
\hline VERECVND & $\begin{array}{c}\text { XII } \\
\text { (DR) }\end{array}$ & & & & & & & I $2(\mathrm{I})$ \\
\hline VINOSE & $\begin{array}{l}\text { XIIII } \\
\text { (MP) }\end{array}$ & $\begin{array}{c}\text { XVIIII } \\
\text { (DE) }\end{array}$ & & & & & & I4 (I) I9 (I) \\
\hline VIX RIDES & $\begin{array}{l}\text { XIII } \\
(\mathrm{PE})\end{array}$ & $\begin{array}{l}\text { XIII } \\
\text { (во) }\end{array}$ & $\begin{array}{l}\text { XIII } \\
\text { (cs) }\end{array}$ & XIII (DE) & & & & I3 (4) \\
\hline$[V A] P P A$ & $\begin{array}{l}\text { VIIII } \\
(\mathrm{PE})\end{array}$ & & & & & & & $9(\mathrm{I})$ \\
\hline NUMERALES & $\begin{array}{l}\text { III A } \\
(\mathrm{MB})\end{array}$ & $\begin{array}{l}\text { VIII A } \\
\text { (MB) }\end{array}$ & $\begin{array}{c}\text { IX } \\
\text { (AP) }\end{array}$ & $\begin{array}{r}\text { XVIII } \\
(\mathrm{PE})\end{array}$ & & & & 3 A (I) 8 A (I) 9 (I) I 8 (I) \\
\hline
\end{tabular}

Fig. 3. Relación de tesserae lusoriae y lugares de hallazgo: (AC) La Alcudia, Mallorca; (AM) Anticuario Mónaco; (AN) AnconaMilán; (AP) Ampurias; (BA) Col. Balboni; (BC) Col. Bruschi; (BM) British Museum; (BO) Col. Bourguignon; (BR) Col. Bruzza; (CB) Col. Borgia; (CG) Col. Guardabassi; (CH) Col. Cherubini; (CL) Museo de Colonia; (CR) Cremona; (CS) Col. Sambón; (CU) Cumas; (DE) Delos; (DR) Col. Dressel; (EL) Alcudia (Elche); (FL) Florencia; (HE) Col. Heckscher; (HR) Col. Hernade; (INC) Col. incierta, Napoli; (IT) Italia; (KA) Col. Kestner; (LO) Col. Lodres; (LV) Museo del Louvre; (мB) Museo de Berlin; (MP) Museo Palermo; (MT) Museo Taranto; (NC) Napoli-Castellani; (NF) Napoli-Fusco; (PE) Perugia; (PO) Pompeya, M. Napoli; (PS) Paestum; (PT) Pentima; (RM) Roma/Marchese Campanari; (RV) Roma, varias colecciones; (TA) Col. Hernández (Tarragona); (TAL) Talamanca, Ibiza; (TOR) Tortosa; (SC) Col. Scalambrini; (SG) Saguntum; (VA) Vaste; (VI) Viterbo.

${ }^{22}$ Podría portar el mismo numeral que en el resto de las tesserae conocidas con la voz Tube, el x. 
se recogen las aparecidas en Hispania. De ahí que hayamos creído conveniente hacer una revisión del total de las piezas publicadas hasta la fecha. El resultado, plasmado en nuestra Fig. 3, ofrece un total de 55 apelativos $^{23}$ que se asocian a los treinta números conocidos.

\begin{tabular}{|l|c|c|}
\hline \multicolumn{1}{|c|}{ DENOMINACIÓN } & N.o $^{\circ}$ DE PIEZAS & CIFRA \\
\hline PERNIX & 9 & XVII \\
\hline TORTVNAT/E & 6 & XXIIII \\
\hline VIX RIDE & 4 & XIII \\
\hline TVBE & 4 & $\mathrm{X}$ \\
\hline EBRIOSE & 4 & $\mathrm{IIII}$ \\
\hline NVGOR & 3 & $\mathrm{I}$ \\
\hline ARGVTE & 3 & $\mathrm{XV}$ \\
\hline
\end{tabular}

FIG. 4. Calificativos asociados siempre al mismo número.

Ahora bien, si nos fijamos cuando el apelativo y el número aparecen suficientemente representados, vemos que la voz Fortunatle es la única que siempre lleva el mismo número, xxiıII, y que esta cifra no aparece en ninguna otra pieza. Esto invita a plantearnos que, aparte de tener un calificativo favorable (afortunado), debía tener un significado y un valor especial en el juego. Por otro lado, la ficha con la expresión Pernix (ágil, veloz) es la más representada hasta el momento, y siempre está ligada al numeral XVII. A continuación se sitúan las que portan los epígrafes Vix Rides, Tube, Ebriose, Nugor o Argute (Fig. 4), todas ellas presentan siempre la misma cantidad, aunque en estos casos el numeral aparece asociado a diversas denominaciones (Fig. 7).

Los nombres más utilizados, aparte de Pernix, son: Vapio, Fur, Felix, Amator, etc. (Fig. 5). Destaca la abundante presencia de tesserae con el término Vapio, que además de utilizar números bajos, se asocia casi en exclusividad con las cifras que cuentan con el nexo 'A' (Fig. 8). No debe ser casual, pues son pocas las que, como veremos, llevan añadida esta letra al numeral. Se pueden plantear varias hipótesis al respecto. Creemos que la presencia de la letra debía darle un valor especial, bien para reafirmar, o bien

23 Serían 57 expresiones si no considerásemos algunas de ellas como una sola, caso de Cinaidus/Cinaedus o Fur/ Fur r[i]de. para cambiar el sentido de la expresión, o incluso el valor del numeral. En el caso de este nombre tal vez fuese una pieza que se emplease como comodín.

\begin{tabular}{|l|c|c|}
\hline \multicolumn{1}{|c|}{ DENOMINACIÓN } & CANTIDAD & NUMERALES \\
\hline PERNIX & 9 & XVII \\
\hline VAPIO & 7 & IIII - IV A - VIII A \\
\hline FORTVNAT/E & 6 & XXIIII \\
\hline FVR/ FVR R $[I] D E$ & 6 & II - II A - III - XIII \\
\hline FELIX & 6 & XII - XXX - XL \\
\hline AMATOR & 6 & XIIII - XXX \\
\hline ARPAX & 5 & XIIII - XVIIII \\
\hline FACETE & 4 & IIII -XI- XXV \\
\hline PATICE & 4 & VII-VIII \\
\hline TVBE & 4 & $\mathrm{X}$ \\
\hline VIX RIDES & 4 & $\mathrm{XIII}$ \\
\hline
\end{tabular}

FIG. 5. Calificativos y numerales más utilizados.

El hecho de que Felix (afortunado, feliz) sea uno de los más representado en las tesserae, no debe extrañarnos, pues, aparte de tener un significado favorable, también porta, entre otras, la cifra más alta del juego, XL. Número que tan sólo comparte con Rex (Fig. 6). Esto nos induce a creer que es posible que sean fichas ganadoras de un premio, o bien el final del juego.

$\mathrm{Al}$ ordenar los valores de mayor a menor (Fig. 6) hemos observado que hay dos series que utilizan los mismos números y términos, con la única salvedad de que una de las listas lleva el nexo 'A' acompañando al numeral. La primera tirada, como se ha apuntado, consta de una sucesión del uno al cuarenta, con la particularidad de que entre el xxx y el XL no hay números. Es paradójico, igualmente, que los números altos: XXVI, XXVIII y XXIX, por el momento, no estén plasmados en ninguna de las tesserae. Es posible que sea producto de la casualidad. No obstante nos llama la atención dicha ausencia, y más teniendo en cuenta el volumen tan amplio de fichas recuperadas, más de 165 .

En otros estudios, la reordenación de números y expresiones ha llevado a algunos investigadores a defender que los números bajos estaban asociados a expresiones desfavorables, mientras que los altos se relacionaban con términos más propicios. En realidad, como vemos en la Fig. 6, no es así, ya que se 


\begin{tabular}{|c|c|c|c|c|c|c|c|}
\hline \multirow{2}{*}{$\begin{array}{c}\text { NÚMERAL } \\
\mathrm{I} \\
\end{array}$} & \multicolumn{6}{|c|}{ NOMBRES/EXPRESIONES } & \multirow{2}{*}{$\begin{array}{c}\mathbf{N}^{\circ}{ }^{\circ} \text { NOMBRES } \\
\end{array}$} \\
\hline & $N V G O R$ & NVGATOR & & & & & \\
\hline II & $F V R$ & & & & & & 1 \\
\hline II A & $\begin{array}{l}F V R / \\
F V R R[I] D E\end{array}$ & STVMACOSE & & & & & 3 \\
\hline III & FVR & MOICE & & & & & 2 \\
\hline III A & MOICE & \begin{tabular}{|l|}
$-\ldots---$ \\
\end{tabular} & & & & & 2 \\
\hline IV & EBRIOSE & FACETE & GVLO & INFELIX & VAPIO & & 5 \\
\hline IIII A & PERIVRE & VAPIO & & & & & 2 \\
\hline $\mathrm{v}$ & CATV...I & FICOSE & & & & & 2 \\
\hline VI & CVNV.LINGVE & $M V L A$ & & & & & 2 \\
\hline VII & CINAIDVS & FICOSE & & & & & 2 \\
\hline VIII & CVNV LINGVE & PATICE & & & & & 2 \\
\hline VIII A & VAPIO & \begin{tabular}{|l|}
$-\ldots--$ \\
\end{tabular} & & & & & 1 \\
\hline $\mathrm{Ix}$ & SCVLTVMO & $P A$ & ---- & & & & 2 \\
\hline $\mathrm{x}$ & CVNNIO & TVBE & & & & & 2 \\
\hline $\mathrm{XI}$ & FACETE & FATVE & GAVDESME & $\angle V P A$ & NVGATOR & TRICO & 6 \\
\hline XI A & TRICO & $\angle V P A$ & & & & & 2 \\
\hline $\mathrm{XII}$ & FELIX & VERECVND & & & & & 2 \\
\hline XII A & FVVCO & & & & & & 1 \\
\hline XIII & $F V R$ & POPINO & VIX RIDES & & & & 3 \\
\hline $\mathrm{XIV}$ & AMATOR & ARPAX & FATVE & FICOSE & MORARIS & VINOSE & 6 \\
\hline $\mathrm{xv}$ & AVIDVS & FACETE & ARGVTE & & & & 3 \\
\hline XVI & LVPA & & & & & & 1 \\
\hline XVII & BONVS & FATVE & PERNIX & & & & 3 \\
\hline XVIII & AVIDVS & PVRVS & \begin{tabular}{|l|}
$-\cdots--$ \\
\end{tabular} & & & & 2 \\
\hline $\mathrm{XIX}$ & ARPAX & SERIVS & VINOSE & & & & 3 \\
\hline $\mathrm{xx}$ & $C L \ldots$ & GVMIA & $P V L$ & & & & 3 \\
\hline $\mathrm{xx} \mathrm{A}$ & BENIGNVS & & & & & & 1 \\
\hline XXI & $A V D A X$ & GVMIA & & & & & 2 \\
\hline XXII & FELIX & & & & & & 1 \\
\hline XXIII & MOECE & & & & & & 1 \\
\hline XXIII A & MALEST & & & & & & 1 \\
\hline xxiv & FORTVNAT/E & & & & & & 1 \\
\hline $\mathrm{xxv}$ & FACETE & BENEST & & & & & 2 \\
\hline XXVI & & & & & & & 0 \\
\hline XXVII & IMPVDES & & & & & & 1 \\
\hline XXVIII & & & & & & & 0 \\
\hline XXIX & & & & & & & 0 \\
\hline $\mathrm{xxx}$ & AMATOR & FELIX & $V E L$ & & & & 3 \\
\hline $\mathrm{xxx} A$ & BENIGNE & FATVE & & & & & 2 \\
\hline $\mathrm{XL}$ & FELIX & $R E X$ & & & & & 2 \\
\hline
\end{tabular}

FIG. 6. Relación entre números y expresiones.

dan ambas opciones. Cecchini (2015: 67) plantea incluso que el número XIII, Vix Rides (finalmente risas), podría haber marcado el límite entre lo positivo y lo negativo, sin que conlleve que sea una regla fija. Sin rechazar de plano esta hipótesis, queremos señalar que hemos comprobado que el número XIII también aparece vinculado con otros términos, como Fur (ladrón) o Popino (glotón). Lo cual nos 


\begin{tabular}{|c|l|l|l|l|l|l|l|}
\hline $\mathrm{XIV}$ & 7 & AMATOR & ARPAX & FATVE & FICOSE & MORARIS & VINOSE \\
\hline $\mathrm{XI}$ & 6 & FACETE & FATVE & GAVDESNE & LVPA & NVGATOR & TRICO \\
\hline $\mathrm{IV}$ & 5 & EBRIOSE & FACETE & GVLO & INFELIX & VAPIO & \\
\hline $\mathrm{XIII}$ & 4 & ARGVTE & FVR & POPINO & VIX RIDE & & \\
\hline $\mathrm{IX}$ & 3 & SCVLTVMO & $\ldots . . P A$ & $\ldots \ldots$ & & & \\
\hline $\mathrm{XVII}$ & 3 & BONVS & FATVE & PERNIX & & & \\
\hline $\mathrm{XVIII}$ & 3 & AVIDVS & PVRVS & $\ldots \ldots$ & & & \\
\hline $\mathrm{XIX}$ & 3 & ARPAX & SERIVS & VINOSE & & & \\
\hline $\mathrm{XX}$ & 3 & CL.... & GVMIA & PVL & & & \\
\hline $\mathrm{XXX}$ & 3 & AMATOR & FELIX & VEL & & & \\
\hline
\end{tabular}

FIG. 7. Relación de los números más utilizados en combinación con los nombres.

\begin{tabular}{|c|c|c|c|}
\hline \multirow{2}{*}{$\frac{\text { NUMERAL }}{\text { II A }}$} & \multicolumn{2}{|c|}{ NOMBRES } & \multirow{2}{*}{$\begin{array}{c}\text { N.o EXPRESIONES } \\
2 \\
\end{array}$} \\
\hline & $F V R / F V R R[I] D E$ (3 veces) & STVMACOSE & \\
\hline III A & MOICE & ------- & I \\
\hline IIII A & PERIVRE & VAPIO (2 veces) & 2 \\
\hline VIII A & VAPIO (4 veces) & ------- & 2 \\
\hline XI A & TRICO & LVPA & 2 \\
\hline XII A & FVVCO (2 veces) & & I \\
\hline $\mathrm{xx}$ A & BENIGNVS (2 veces) & FATVE & 2 \\
\hline XXIII A & MALEST & & $\mathrm{I}$ \\
\hline $\mathrm{xxx} A$ & BENIGNE & & I \\
\hline
\end{tabular}

Fig. 8. Relación de numerales acompañados de la letra a y de calificativos.

plantea serias dudas poder aceptar que realmente esta cifra definiera ese listón que separa lo benévolo de lo desfavorable.

Los números más empleados, siguiendo este orden, son el XIV, el XI, el IV y el XIII (Fig. 7). La mayoría de ellos aparecen asociados a calificativos soeces como: adúltero, ladrón, prostituta, impostor, borracho, embaucador, sufridor de hemorroides, etc. Esto indica que, al haber un mayor número de fichas con esos términos, tendrían más posibilidades de salir en el hipotético 'juego'.

Cecchini (2015: 67-68), al encontrarse con tesserae en las que aparece junto al número la letra 'A' 24 , plantea la existencia de dos series diferenciadas: una principal y otra secundaria. Creemos que, aunque esto es plausible, ambas debían complementarse y formar parte de un único juego, en el que el valor de los números sería modificado cuando apareciese acompañado de la letra 'A', bien para

24 En algunas publicaciones aparece como 'AL'. Huelsen (1896: 227 y ss.) transcribe como 'A'. sumar y darle más valor a la ficha, o bien para conseguir el efecto contrario al que marcaba, reducir puntos, acciones, etc.

Este nuevo repertorio que presentamos (Fig. 8) se compone, por el momento, de 9 tesserae lusoriae con valores que van desde el II al xxx. No encontramos por el momento ninguna correlación entre los números y los calificativos, tan sólo llegamos a ver números sucesivos entre el ir y el IIII. A partir de ahí se dan aleatoriamente (Fig. 8). En el caso de los nombres la mayoría se corresponden con expresiones desfavorables, siendo minoritarios los benévolos. Algunos se repiten dos o tres veces, salvo el ya mencionado de Vapio, que aparece en cuatro ocasiones con el numero viII y en dos con el IV. Podría implicar, como apuntamos en su momento, que estemos ante una pieza empleada como comodín.

Para concluir, podemos apuntar que, a través de las diversas tablas que hemos realizado, nos damos cuenta de que en muy pocas ocasiones las expresiones más utilizadas se asocian al mismo número. 
Es el caso de los calificativos Pernix (xviI), Tube (x), Ebriose (III), Fortunat (xxIIII) o Vix Ride (xIII), siendo lo más habitual que haya voces asociadas a varios números. Vemos, además, que indistintamente unos tienen carácter negativo y otros positivo. Nos llama poderosamente la atención que el número xxiII esté reservado únicamente para el apelativo Fortunat, lo cual no puede ser casual.

Hemos observado tras la correlación realizada en la Fig. 4 que hay dos listas de números, cuya única diferencia está en la 'A' añadida al numeral. Estamos de acuerdo con Cecchini (2015: 67 y ss.) en que se trata de dos series, aunque discrepamos en que sean independientes, pensamos que ambas interactuaban a la vez en el juego.

Pese a contar con todos estos datos, no encontramos una solución lógica que pueda inducirnos a comprender las normas que regulaban este juego. Es preciso que nuevos hallazgos vayan rellenando las seriaciones conocidas y, de este modo, poder hacer planteamientos más precisos sobre las correlaciones de nombres y expresiones.

Por último, hay que señalar, para el caso hispano, la presencia masiva de este modelo de tesserae exclusivamente en el arco catalano-levantino-balear. Cuestión que, como proponemos, se debe a conexiones ancestrales con la península italiana.

\section{Bibliografía}

Abascal, J. M. (2012): "Cinco inscripciones del sur del conventus Carthaginensis (Hispania Citerior)", Zeitschrift für Papyrologie und Epigraphik, 183, pp. 277281.

Albertini, E. (1918): "Inscriptions d'Espagne", Mélanges d'Archéologie et d'Histoire, 37, pp. 309-331.

Alföldy, G.; Clauss, M. y Mayer, M. (1995): Corpus Inscriptionum Latinarum II, Pars XIV. Conventus Tarraconensis. Pars Meridionalis. Berlin.

Almagro Bach, M. (1952): Las inscripciones ampuritanas griegas, ibéricas y latinas. Barcelona.

Andreau, J. (2001): "Deux tessères nummularies inédites", Revue Numismatique, 6, n. ${ }^{\circ} 157$, pp. 329-336.

Balil, A. (1987): "Tesserae lusoriae de Ampurias", Archivos de Prehistoria Levantina, 17, Homenaje a D. Fletcher, pp. 331-336.

(C) Universidad de Salamanca
Baratta, G. (2014): "Le tesserae lusoriae delle Isole Baleari”. En Catalina Ferrando, C. y Costa, B. (eds.): In Amicitia. Miscel.làni d'estudis en Homenatge a Jordi H. Fernández. Ibiza, pp. 69-74.

Beltrán, A. (1983): "La vida de Pollentia según la epigrafía”. En Ripoll, P.: Symposium Pollentia y la Romanización de las Baleares (La Alcudia 1977). Mallorca, pp. 47-53.

Blanchet, I. A. (1889): "Tessères Antiques. Téâtrales et autres", Revue Archéologique, serie III, n. ${ }^{\circ}$ XIV (1), pp. 225-242 y 369-380.

Campagna, L. (1995): "Cisterne e buca di scarico di età repubblicana a Vaste (Le), scavi di fondo S. Antonio", Studi Antichitè, 8 (2), pp. 215-288.

Casagrande, M. (2012): "Le sortes di Perugia". En Piranomonte, M. y Simón, F. M. (eds.): Contesti Magici/Contextos Mágicos (Atti del Convegno Internazionale). Roma, pp. 247-252.

Castelló, J. J. (1988): Epigrafía romana de Ebusus. Ibiza.

Cecchini, N. (2015): “Tessere parallelepipede". En Lambrugo, Cl.; Slavazzi, F. y Fedeli, A. M. (eds.): I materiali della collezione Archeologica "Giulio Sambon" di Milano. 1. Tra alea e agón: giochi di abilità e di azzardo. Firenze, pp. 67-70.

Cecchini, N.; Bianchi, Ch. y Diani, M. G. (2015): "Le scoperte di Cremona". En Lambrugo, C.; Slavazzzi, F. y Fedeli, A. M. (eds.): I materiali della collezione Archeologica "Giulio Sambon" di Milano. 1. Tra alea e agòn: giochi di abilità e di azzardo. Firenze, pp. 121-126.

Comes, R. y RodÀ, I. (eds.) (2002): Scripta manent: la memoria escrita dels romans. Catálogo de la Exposición. Barcelona: MAC.

Corell, J. (1993): "Els jocs d'atzar en l'epigrafia romana de Sagunt", Braçal, 8, pp. 45-50.

Corell, J. (2002): Inscripcions romanes del Pais Valencià IA (Saguntum I. El seu territorio). Fonts històriques valencianes). Valencia: Univ. de Valencia.

De Grossi, J. (2011): Artigiani dell'osso, avorio e palco. Ornamenti, utensili e giochi dalla prehistoria al medioevo. Lecce.

Deona, W. (1938): Exploration archéologique de Délos 18. Le mobilier délien. Paris.

Deschler-Erb, S. y Gostenčnik, K. (2008): “Différences et identités de la vie quotidienne dans les provinces romaines: l'exemple de la tabletterie". En Bertrand, I. (ed.): Le travail de l'os, du bois de cerf et de la corne à l'époque romaine: un artisanat en marge? Monographies Instrumentum, 34. Montagnac, pp. 283-309. 
FABre, G.; MaYer, M. y RodÀ, I. (1984): Inscriptions romaines de Catalogne, I: Barcelone (sauf Barcino). Paris: Diffusion de Brocard.

FABRE, G.; MAYER, M. y RodÀ, I. (2002): Inscriptions romaines de Catalogne, v: Suppléments aux volumes I-IV et instrumentum inscriptum. Paris: Diffusion de Brocard.

Fita, F. (1907): "Antiguedades ebusitanas", Boletín Real Academia de la Historia, 51, pp. 322-384.

Gostenčnik, K. (2005): Die Beinfunde vom Magdalensberg (Archäologische Forschungen zu den Grabungen auf dem Magdalensberg 15; Kärntner Museums-schiften, 78). Klagenfurt.

Graillot, H. (1896): "Une collectios de tessères", Mélanges d'Archéologie et d'Histoire, 16, pp. 299-314.

Huelsen, Ch. (1896): "Miscellanea epigrafica", Mitteilungen des Kaiserlich Deutschen Archaeologischen Institus, Römische Abteilung, xI, pp. 227-257.
Mayer, M. y RodÀ, I. (1985): "Consideraciones sobre el conjunto epigráfico de Dertosa”. En Actas CAN XVII (Logroño 1983). Zaragoza, pp. 701-737.

Mayer, M. y Rodì, I. (1988): "Epigrafía", Fonaments, 7, pp. 194-263.

Pedroni, L. (1995): “Tessere da una collezione privata”, Archeologia Classica, XLviI, pp. 161-197.

Puig i Cadafalch, J. (1936): La catedral visigótica d'Egara. Anales del Instituto de Estudios Catalanes, vi. Barcelona.

Veny, C. (1965): Corpus de las inscripciones baleáricas hasta la dominación árabe. Madrid.

Zeis, C. (1921). "V. Ferento (Viterbo) Scoperta di tombe di età repubblicana", Notizie degli Scavi Antichità, fasc. 7-9, pp. 215-228. 